\title{
Evaluation of the Physicochemical Behavior of Waste Water Treatment Polyelectrolytes with Metal Ions
}

\author{
Eduardo A. López Maldonado ${ }^{1}$, Mercedes T. Oropeza Guzmán ${ }^{1,2}$, Georgina Pina-Luis ${ }^{1}$, \\ Adrián Ochoa-Terán ${ }^{*}$ \\ ${ }^{1}$ Graduate and Research Center of the Technical Institute of Tijuana, Tijuana, Mexico; ${ }^{2}$ Centre for Research and Technological De- \\ velopment in Electrochemistry, Tijuana, Mexico. \\ Email: *ochoa@tectijuana.mx
}

Received November $25^{\text {th }}, 2012$; revised January $3^{\text {rd }}, 2013$; accepted February $2^{\text {nd }}, 2013$

\begin{abstract}
In this paper is describing the physicochemical behavior of polyelectrolytes (PEs) used in waste water treatment with mono-, di- and trivalent metal ions as $\mathrm{K}^{+}, \mathrm{Mg}^{2+}, \mathrm{Zn}^{2+}, \mathrm{Fe}^{3+}, \mathrm{Sn}^{2+}, \mathrm{Cd}^{2+}, \mathrm{Pb}^{2+}, \mathrm{Cu}^{2+}, \mathrm{Ni}^{2+}, \mathrm{Al}^{3+}$ and $\mathrm{Cr}^{3+}$. A coagulant polyelectrolyte Poly(vinyl sulfate) potassium salt (PVSK), and a commercial available Flocculant Trident 2756, were used as models for the study. The colloidal titration UV-Vis spectroscopy technique was successfully implemented in order to evaluate the complexation of PEs with Toluidine Blue O (OTB) and the ability of different metal ions to displace the OTB from the PE-OTB complex and form the PE-metal ion complex. From the experiments was concluded that PVSK has a high affinity for $\mathrm{Al}^{3+}$ and $\mathrm{Mg}^{2+}$ while the Flocculant has the highest affinity for $\mathrm{Sn}^{2+}$ followed by $\mathrm{Zn}^{2+}$ and $\mathrm{Mg}^{2+}$. The absorbance profiles of polyelectrolyte-OTB complex (Absorbance vs. Metal/PE) were used to calculate association constants. On the other hand, the mass balance of OTB and its absorbance profiles were used to calculate the association constants of polyelectrolyte-metal ion complexes. Thus metal ions with the highest affinities have the highest association constant. Metal ions with the highest affinities present the highest values of association constant.
\end{abstract}

Keywords: Polyelectrolytes; Metal Ions; Colloidal Titration; UV-Vis Spectroscopy; O-Toluidine; Wastewater Treatment

\section{Introduction}

Polyelectrolyte chains have atoms or groups of atoms which have the ability to chelate or exchange sities with metal ions [1]. The most common coordinating atoms present in a polymer chain, either central or side chain, are nitrogen, oxygen, phosphorus and sulfur [2]. For those polymers in which the polymer-metal ion interaction involves an ion exchange process, the most common are the quaternary ammonium with chloride, sulphate and hydroxyl counterions [3]. Polyelectrolyte-metal ion interaction could be electrostatic or may include the formation of coordinated bonds. The type of interaction depends on the chemical nature (ionization potential and electronic affinity) of the functional groups. The variables that affect the PE-metal ion interaction are classified into two groups: intrinsic and extrinsic to the polymer [4]. The former group includes the polymer structure in terms of composition and geometry, which affects the flexibility of the chains in solution: branches of the chain, chemical nature of the functional groups, and their dis-

\footnotetext{
${ }^{*}$ Corresponding author.
}

tribution at the polymer chain, etc. The second group includes the charge and type of the metal ion, $\mathrm{pH}$, ionic strength, temperature and dielectric constant of the medium $[5,6]$. The knowledge of association phenomena of metal ions with charged macromolecules is essential for understanding of their physicochemical behavior in environmental, biological systems and in waste water treatment by coagulation-flocculation [7-9].

Polyelectrolytes are used in coagulation-flocculation processes mainly to remove suspended particles, turbidity and color. However, due to their chemical nature, polyelectrolytes also interact with oppositely charged species as metal ions that are present in the wastewater. For example, the treatment of waste water from semiconductor fabrication industry with high suspended solids content of heavy metals, where the objective is to remove suspended solids as well as heavy metals content. Therefore, it is important to study the physicochemical behavior of polyelectrolytes with different metal ions present in the waste water, with the aim of determining the affinity and capacity of removal of each metal with polyelectrolytes, in order to determinated the optimal 
dose of required for maximum removal of heavy metals.

The study of the PE-metal ion interaction can be carried out by different analytical techniques such as potentiometry [5-8], spectrophotometry [9-12], viscosity [10, $13]$, ionic conductivity [14], light scattering [15,16], voltammetry [17,18], liquid-phase polymer-based retention (LPR) [19,20] and fluorescence [21-24]. Colloidal titration is a conventional method used in determination of cationic polyelectrolytes concentration; the PVS-OTB complex is used to detect the equivalence point of the titration [25]. In literature there are no reports where PE-OTB complex is used to determine the affinity of coagulants and flocculants to heavy metals in water. In this paper we present our results regarding to the study of the physicochemical behavior of polyelectrolytes used in residual water treatment for coagulation-flocculation processes with metal ions, based on the displacement of OTB in PE-OTB complexes by metal ions.

\section{Materials and Methods}

\subsection{Reagents}

Poly (vinyl sulfate) potassium salt (PVSK) with molar mass of $170 \mathrm{KDa}$, was obtained from Sigma-Aldrich. The negative charges are attributed to sulfate group in each monomer. Flocculant (Trident 27506) that are commercial polyelectrolyte intended to be quantified in an industrial waste water treatment process. Concentration of PVSK is expressed in $\mathrm{mg} / \mathrm{L}$ in order to compare with results obtained for Flocculant and also in molar concentration (mM) expressed on the basis of a monomeric unit which indicates the moles of ionic group per liter of the polymer in solution.

As the structure and identity are unknown for Flocculant concentration is expressed in $\mathrm{mg} / \mathrm{L}$.

Toluidine Blue $\mathrm{O}$ solution was used as an indicator and was obtained from Sigma-Aldrich. Aluminum, cadmium, chromium, copper, iron, potassium, nickel, lead, tin, zinc and magnesium standard solutions was obtained for AAS at $1000 \mathrm{mg} / \mathrm{L}$ (FLUKA).

\subsection{Apparatus}

Absorbance of solutions was measured at $400-800 \mathrm{~nm}$ with a UV-Vis spectrophotometer (Cary 100 Conc, Varian) using a $10 \mathrm{~mm}$ pathlength cell.

pH Meter was a Denver Instrument UP-5.

The FTIR spectra were recorded on a Spectrum 400 FT-IR/FT-NIR spectrometer (Perkin Elmer).

\subsection{Determination of the Optimal Dose of OTB}

Synthetic PVS-OTB solutions were prepared mixing 1.0 $\mathrm{mL}$ of a $201.1 \mathrm{mg} / \mathrm{L}(1.24 \mathrm{mM})$ PVSK solution and different aliquots of a $82.6 \mathrm{mg} / \mathrm{L}(0.27 \mathrm{mM})$ OTB solution graduated to a $10 \mathrm{~mL}$ final volume.

The optimal OTB/PVSK ratio was determinated according to the following procedure: Solutions were prepared mixing $1.0 \mathrm{~mL}$ of a $201.1 \mathrm{mg} / \mathrm{L}$ PVSK solutions and different aliquots from 0.1 to $1.0 \mathrm{~mL}$ of $82.6 \mathrm{mg} / \mathrm{L}$ OTB solution and diluted to $10 \mathrm{~mL}$ final volume with deionized water. A solution was prepared with $1 \mathrm{~mL}$ of $82.6 \mathrm{mg} / \mathrm{L} \mathrm{mM} \mathrm{OTB}$ and diluted to $10 \mathrm{~mL}$ with deionized water; this solution was taken as a blank. UV-Vis absorbance of each solution was recorded and a graph of absorbance at $509 \mathrm{~nm} v \mathrm{~g}$ g OTB was constructed. The same methodology was used to determine the optimal dose of OTB in the study of Flocculant-OTB interaction. The ignition concentration of Flocculant was $22.4 \mathrm{mg} / \mathrm{L}$.

\subsection{Study of Interaction Anionic PE-Metal Ion by UV-Visible}

All titrations were performed in a batch model. The solutions were prepared mixing $1.0 \mathrm{~mL}$ of a $201.1 \mathrm{mg} / \mathrm{L}$ KPVS solution (1.24 $\mu$ moles) and $0.5 \mathrm{~mL}$ of a 82.6 $\mathrm{mg} / \mathrm{mL}$ OTB solution $(0.135 \mu$ moles $)$ with different aliquots of metal ion solutions such as $\mathrm{Pb}^{2+}, \mathrm{Fe}^{3+}, \mathrm{Cd}^{2+}, \mathrm{Cr}^{3+}$, $\mathrm{Cu}^{2+}, \mathrm{K}^{+}, \mathrm{Mg}^{2+}, \mathrm{Ni}^{3+}$ and $\mathrm{Sn}^{2+}$ at $10 \mathrm{mg} / \mathrm{L}$. Then, UV-Vis spectra were obtained for all samples. The same procedure was followed with Flocculant, mixing $1.0 \mathrm{~mL}$ of a $22.4 \mathrm{mg} / \mathrm{L}$ solution with $0.5 \mathrm{~mL}$ of a $82.6 \mathrm{mg} / \mathrm{L}(0.27$ $\mathrm{mM}$ ) OTB solution with different aliquots of metal ion solutions $10 \mathrm{mg} / \mathrm{L}$.

\section{Results and Discussions}

The experimental strategy was based on use PE-OTB complexes such as PVS-OTB and Flocculant-OTB to study the interaction of the metal ions with polyelectrolyte. First, a colored complex is formed between the anionic polyelectrolyte and OTB and then is titrated with a metal ion to effect the displacement of the colorant (Figure 1). The displacement of OTB was follow to determine indirectly the affinity between polyelectrolytes and metal ions. From displacement profiles were calculated relative association constants of the PE-metal ions complexes.

\subsection{Study of Interaction PVS-Metal Ion by UV-Vis}

First, was determinate the optimal relationship between PVSK and OTB for the study. As can be seen in Figure 2, free OTB has a maximum of absorbance at $628 \mathrm{~nm}$ and decreases when OTB interacts with PVSK. The PVS-OTB complex formed by the association of these two species presents an absorbance band at $509 \mathrm{~nm}$. In the first solution $(0.20 \mathrm{mg}$ of PVSK $+8.26 \mu \mathrm{g}$ of OTB) the spectra shows almost exclusively the absorption band corresponding to the PVS-OTB complex at $509 \mathrm{~nm}$. For 


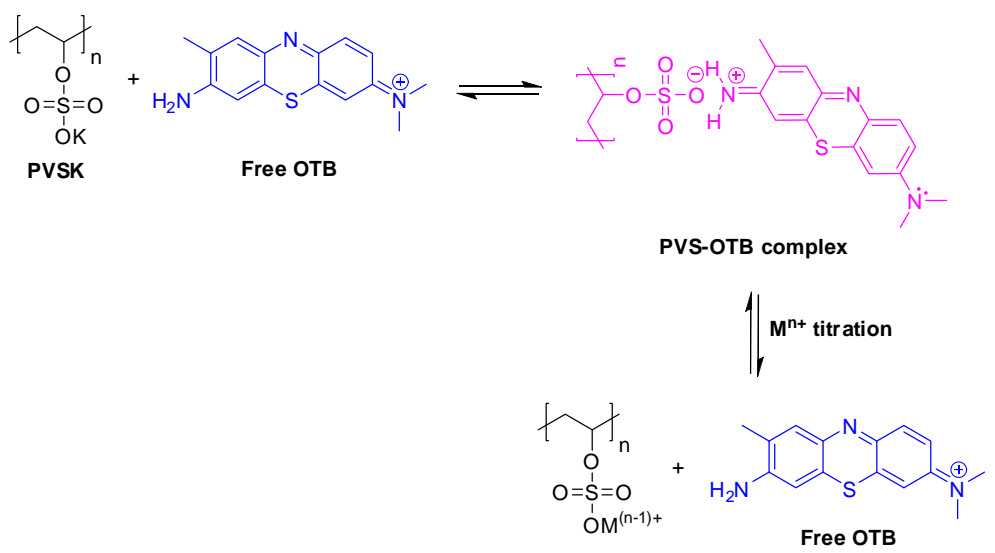

Figure 1. Schematic representation for the study the PE-metal ions interactions.

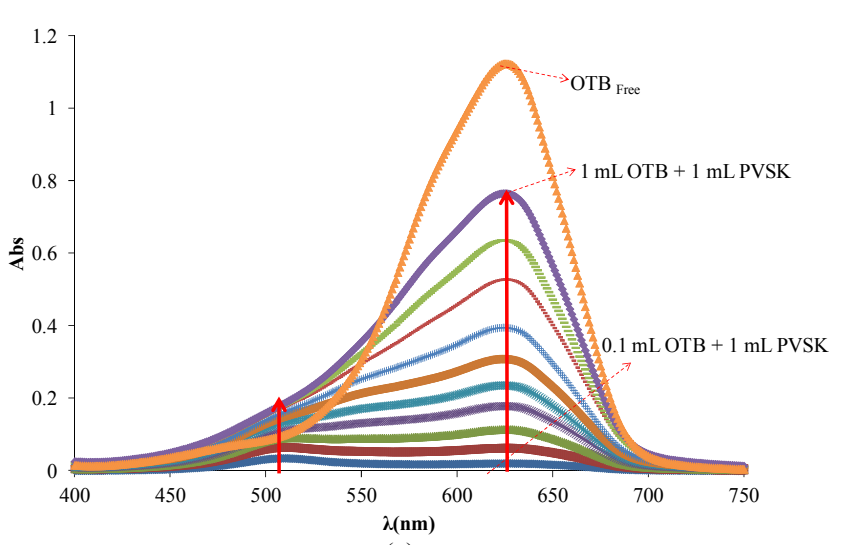

(a)

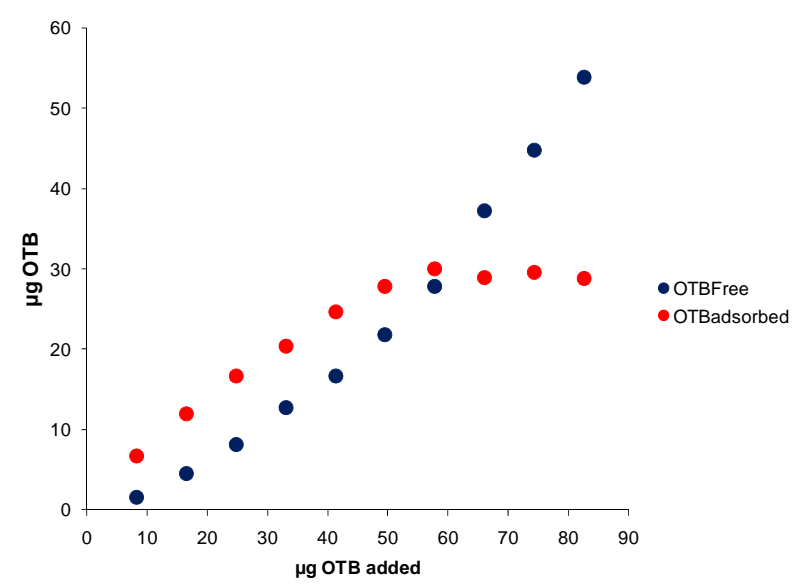

(b)

Figure 2. (a) Absorbance spectra of solutions containing $0.20 \mathrm{mg}$ of PVSK with increasing additions of 8.26 $\mu \mathrm{g}$ of OTB; (b) Bound and free OTB amount profile versus total OTB added.

the rest of combinations the absorption band corresponding to the complex increases, but also the absorption band of free OTB indicating that at higher OTB concentration, PVSK has been saturated.

In Figure 2(b) are shown the bound and free OTB amount profiles versus the amount of OTB added. It is observed a change in slope at $50 \mu \mathrm{g}$ of OTB added. Beyond this point the amount of bound OTB remains constant and free OTB increases gradually. This corresponds to the equivalence point titration of PVSK with OTB. In order to eliminate variations due to saturation phenomena was selected the amount of $41.30 \mu \mathrm{g}$ OTB added $(0.135$ $\mu$ moles OTB $/ 1.24 \mu$ moles PVSK) for the titration study with metal ions. Also, at this relationship there are 24.65 $\mu \mathrm{g}(0.080 \mu \mathrm{mol})$ of bound OTB and $14.65 \mu \mathrm{g}(0.048$ $\mu \mathrm{mol})$ of free OTB.

When $\mathrm{K}+$ is exchanged by OTB on the PVS the solution has a pale pink coloration, but as the metal is added, OTB colorant is released and the solution turn to a blue, the typical color that indicates the presence of free OTB (Figure 3). In the absorbance spectra was observed that

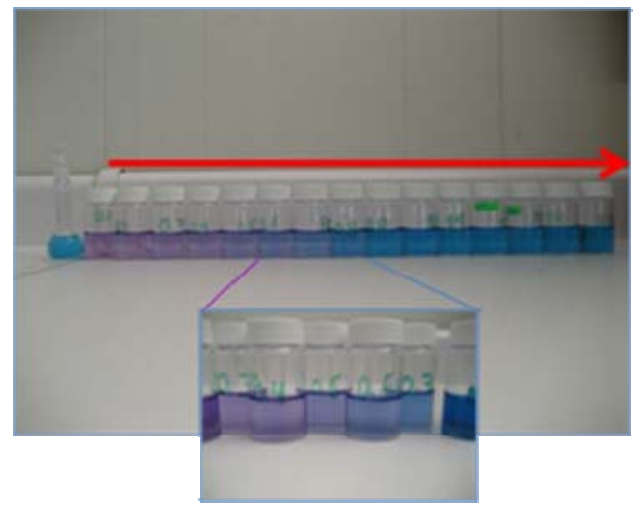

Figure 3. Release of OTB in titration of anionic PE with metal ions.

as the metal ion amount increases, the absorbance band at $509 \mathrm{~nm}$ corresponding to the PVS-OTB complex gradually decreases and the absorbance band at $628 \mathrm{~nm}$ increases.

The UV-Vis spectra obtained in the titration of PVSOTB complex with $\mathrm{Al}^{3+}$ and $\mathrm{Pb}^{2+}$ are shown in Figure 4. 


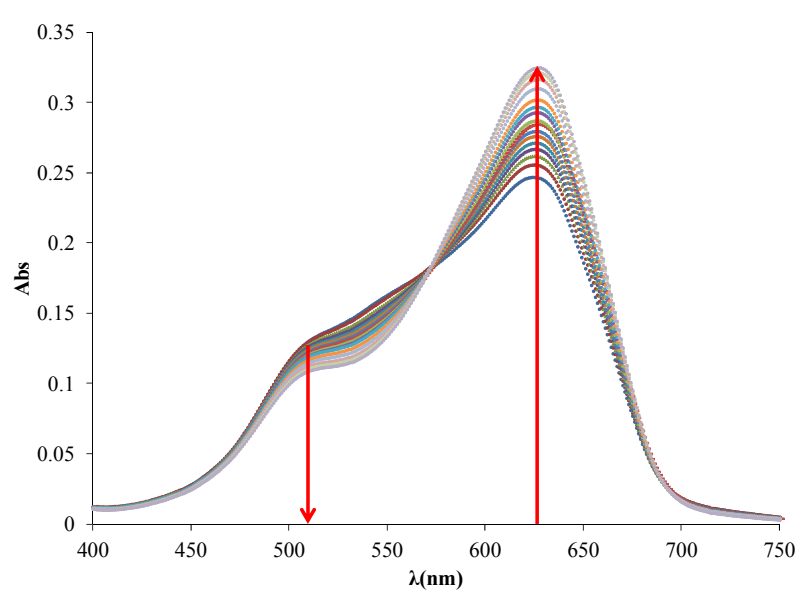

(a)

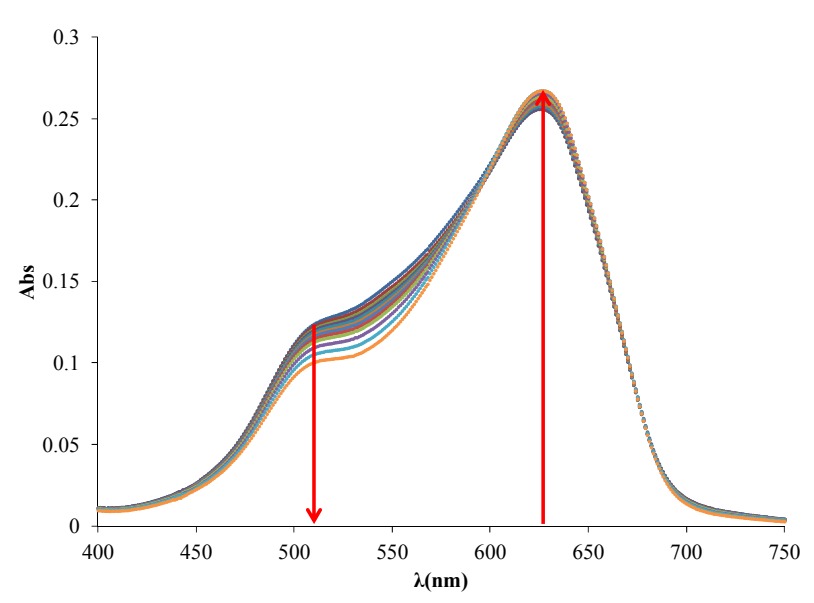

(c)

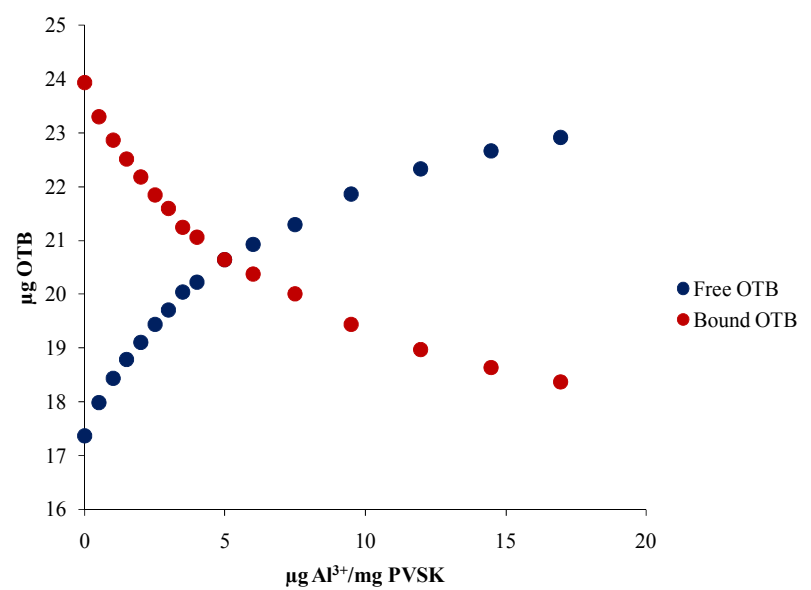

(b)

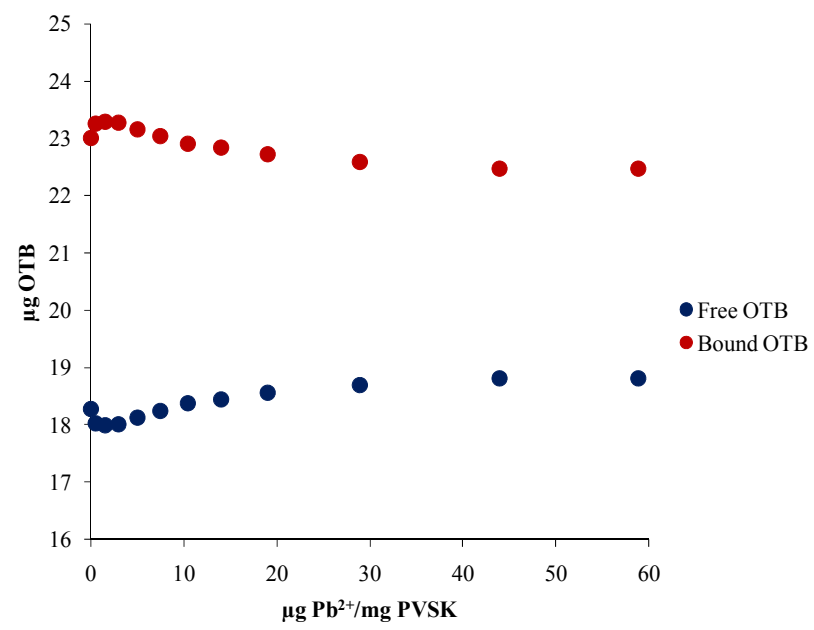

(d)

Figure 4. UV-visible spectra obtained by titration of PVSK-OTB complex with $\mathrm{Al}^{3+}$ and $\mathrm{Pb}^{2+}$ (a) and (c) and bound and free OTB amount profiles (b) and (d).

It is noteworthy that $\mathrm{Al}^{3+}$ causes a greater increase than $\mathrm{Pb}^{2+}$ in the absorption band at $628 \mathrm{~nm}$ because the higher displacement of the OTB complexed to the anionic polyelectrolyte. Another significant difference is the location of the isosbestic points, which are located at 573 $\mathrm{nm}$ for $\mathrm{Al}^{3+}$ and at $603 \mathrm{~nm}$ for $\mathrm{Pb}^{2+}$ complexes.

The bound and free OTB amount profile obtained by titration of PVS-OTB showed that at $17 \mu \mathrm{g}(0.628 \mu \mathrm{mol})$ of $\mathrm{Al}^{3+} / \mathrm{mg}$ PVSK, $5.55 \mu \mathrm{g}(0.018 \mu \mathrm{mol})$ of OTB were released.

With the same amount of $\mathrm{Pb}^{2+}$ only $0.28 \mu \mathrm{g}(0.0009$ $\mu \mathrm{mol})$ of OTB were displaced by the metal ion. In this sense, PVS has twenty times more affinity for $\mathrm{Al}^{3+}$ than $\mathrm{Pb}^{2+}$.

The absorbance profiles obtained by titration of PVSOTB complex with various metal ions are shown in Figure 5. In general, is possible to distinguish three groups according to the affinity that presents each metal ion with the anionic polyelectrolyte. Metal ions $\mathrm{Al}^{3+}, \mathrm{Mg}^{2+}$ and
$\mathrm{Cr}^{3+}$ are the group with more affinity. The second group corresponds to $\mathrm{Zn}^{2+}, \mathrm{Sn}^{2+}$ and $\mathrm{Fe}^{3+}$ having intermediate affinity, and $\mathrm{Ni}^{2+}, \mathrm{Cd}^{2+}, \mathrm{K}^{+}, \mathrm{Cu}^{2+}$ and $\mathrm{Pb}^{2+}$ are the metal ions with the lower affinity. The OTB release ratio depends on the concentration, number of valence and affinity of polyelectrolyte toward the metal ion.

Observed affinity can be explained in terms of the well known Hard and Soft Acids and Bases principles (HSAB) proposed by Pearson.

Sulfate anion is a hard base and has more affinity toward hard acids such as $\mathrm{Al}^{3+}, \mathrm{Mg}^{2+}$ and $\mathrm{Cr}^{3+}$; an intermediate affinity for semihard acids as to $\mathrm{Zn}^{2+}$ and $\mathrm{Fe}^{3+}$ and lower affinity toward soft acids as $\mathrm{Pb}^{2+}$.

Relationships metal ion/PE were variable due to metal ions with greater affinity favored formation of flocs or suspended solids at very low doses (17 $\mu \mathrm{g} \mathrm{Al}^{3+} / \mathrm{mg}$ PVS), this is related to the effect of the metal ion valence because polyvalent ions can form ionic bonds with more than one anionic monomer unit of PVS and this promotes 


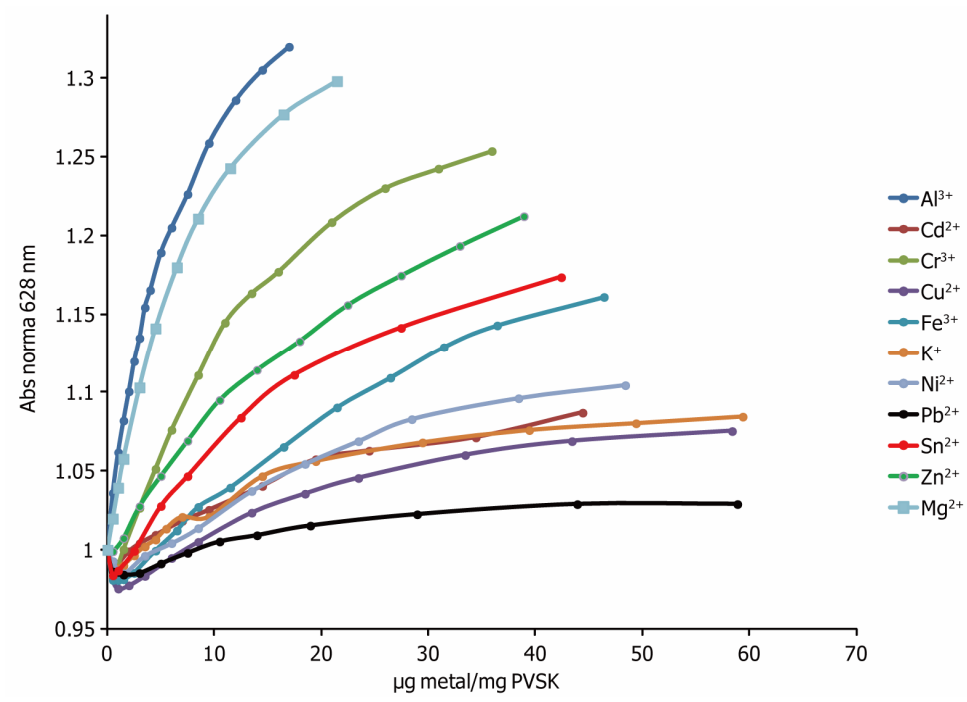

Figure 5. Normalized absorbance profiles obtained by titration of PVS-OTB and various metal ions.

the formation of flocs (see Figure 6), whereas with lower affinity ions higher ratios were achieved without presenting formation of solids or significant changes in absorbance even at relationships $\mu \mathrm{g}$ metal/mg PVSK equal to 60 .

Equations (1) and (2) represent the equilibrium between PVSK and OTB to form the PVSK-OTB complex and the equilibrium in which the PVS-OTB complex is used to study the physicochemical behavior of anionic polyelectrolyte by exchange of OTB with metal ions. These two processes are in competition and are related with association constants $\mathrm{K}_{1}$ and $\mathrm{K}_{2}$, respectively. As was described, the amount of displaced OTB by metal ion depends on the affinity between polyelectrolyte and cation. The magnitude of $\mathrm{K}_{2}$ is a relative measure of this affinity. In summary, metal ions which exhibit higher $\mathrm{K}_{2}$ values should effect a greater displacement of OTB.

$$
\begin{aligned}
\operatorname{PVSK}(-)+ & \mathrm{OTB}(+) \stackrel{\mathrm{K}_{1}}{\rightleftarrows}[\mathrm{OTB}(+) / \operatorname{PVSK}(-)] \\
& {[\mathrm{OTB}(+) / \operatorname{PVSK}(-)]+\text { Metal } } \\
& \stackrel{\mathrm{K}_{2}}{\rightleftarrows}[\operatorname{PVSK}(-) / \text { Metal }]+\mathrm{OTB}_{\text {Free }}
\end{aligned}
$$

The association constant $\mathrm{K}_{1}$ for PVSK-OTB complex was determined from the absorbance data profile at 509 $\mathrm{nm}$, while the association constants of the PVS-metal ion complex were calculated indirectly using absorbance profiles at $628 \mathrm{~nm}$. Calculation of the constants was performed by a curve fitting based on a non-linear leastsquares method [26] assuming that each monomeric unit of PVS interacts with a metal ion forming a 1:1 complex. This assumption is done considering that the equilibrium are achieved before the flocs formation. In Table $\mathbf{1}$ are reported the values obtained and is noteworthy that metal ions performing a higher displacement of OTB in PVS-OTB complex yields the greater magnitudes for $\mathrm{K}_{2}$.

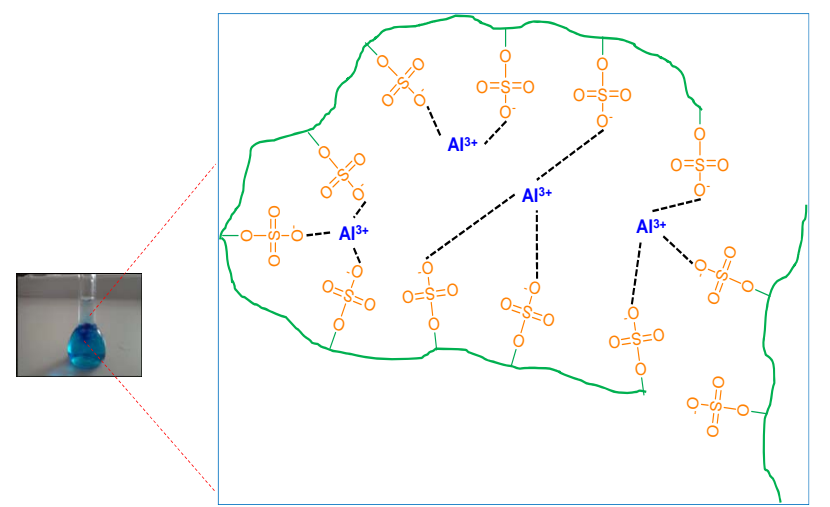

Figure 6. Floc formation with metal ions with $\mathrm{Al}^{3+}$ and PVS at ratios higher than $17 \mu \mathrm{g}$ metal ion/mg PE.

Table 1. Relative association constants of metal ions and PVSK.

\begin{tabular}{cc}
\hline Cation & $\mathbf{K}(\mathbf{m L} / \mathbf{\mu g})$ \\
\hline $\mathrm{Al}^{3+}$ & 811.04 \\
$\mathrm{Mg}^{2+}$ & 718.14 \\
$\mathrm{Cr}^{2+}$ & 357.40 \\
$\mathrm{~K}^{+}$ & 279.92 \\
$\mathrm{Sn}^{2+}$ & 236.00 \\
$\mathrm{~Pb}^{2+}$ & 190.26 \\
$\mathrm{Cd}^{2+}$ & 180.22 \\
$\mathrm{Cu}^{2+}$ & 142.70 \\
$\mathrm{Zn}^{2+}$ & 139.22 \\
$\mathrm{Ni}^{2+}$ & 90.83 \\
$\mathrm{OTB}^{3+}$ & 53.18 \\
$\mathrm{Fe}^{3+}$ & 52.38 \\
\hline
\end{tabular}


Moreover, metal ions that generate less displacement OTB have lower $\mathrm{K}_{2}$ values or even near the constant $\mathrm{K}_{1}$.

Here, is interesting that association constant of PVS$\mathrm{K}^{+}$complex is greater than PVS-OTB constant, this is the reason that OTB promotes low displacement of $\mathrm{K}^{+}$in PVSK and when PVS-OTB complex is retitrated with $\mathrm{K}^{+}$ the latter displaces OTB.

\subsection{Study of Interaction Flocculant-Metal Ions by UV-Visible}

The optimal relationship between Flocculant and OTB was determinate for the study. In Figure 7 free OTB band has a maximum absorbance at $628 \mathrm{~nm}$ and decreases when OTB interacts with Flocculant. In the first solution $(0.224 \mathrm{mg}$ of Flocculant $+8.26 \mu \mathrm{g}$ of OTB) the spectrum shows an absorption band corresponding to the Flocculant-OTB complex at $555 \mathrm{~nm}$. For the rest of combinations the absorbance increases as well as the absorbance of free OTB indicating that at higher OTB concentrations, Flocculant has not been saturated.
In Figure 7(b) is shown the bound and free OTB profile versus the total amount of OTB added. It is observed a slight change in slope at $50 \mu \mathrm{g}$ of OTB added. This might correspond to the equivalence point titration, but unlike PVSK, Flocculant does not saturate at higher amounts of OTB. Then, the selected ratio for the titration study was $41.3 \mu \mathrm{g}$ (0.135 $\mu$ moles) OTB/0.2236 mg Flocculant. At this ralationship there are $22.9 \mu \mathrm{g}(0.075$ $\mu \mathrm{mol})$ of bound OTB and $18.4 \mu \mathrm{g}(0.060 \mu \mathrm{mol})$ of free OTB.

Also, was studied the effect of $\mathrm{pH}$ on the FlocculantOTB interaction due to the composition and structure of Flocculant is unknown. As is shown in Figure 8 the interaction Flocculant-OTB is dependent of $\mathrm{pH}$. At lower $\mathrm{pH}(2-4)$ is observed the highest absorbance for free OTB. Beyond a $\mathrm{pH} 4$ appears the absorbtion band at 555 of Flocculant-OTB complex. At the same time, the absorbance at $628 \mathrm{~nm}$ decreases into the range of $\mathrm{pH} 4-6.5$ and remain constant at higher pHs. These results demonstrated that at low $\mathrm{pH}$ the functional groups in the Floc-

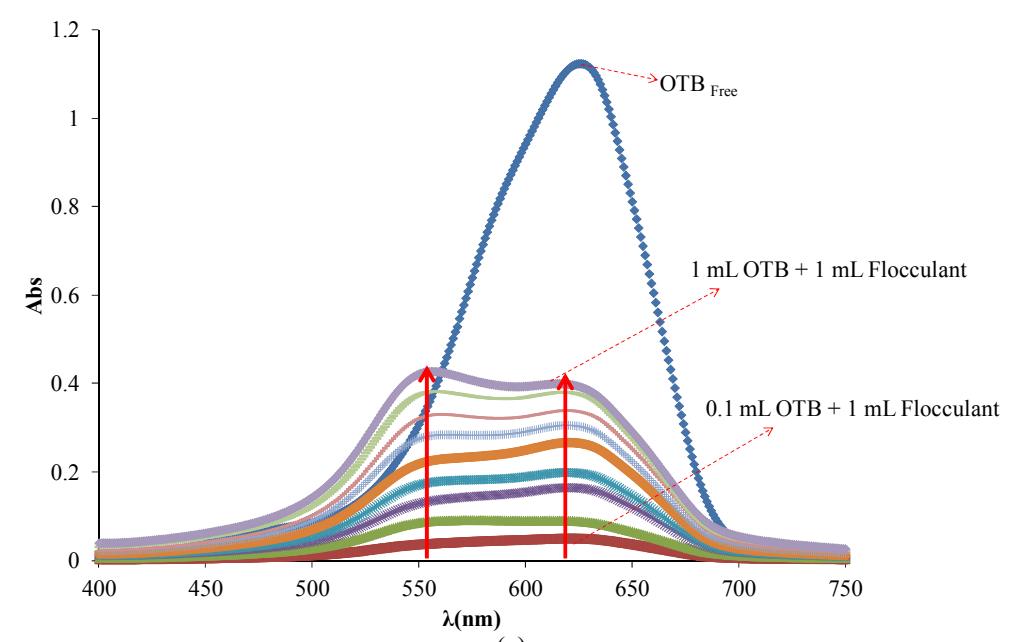

(a)

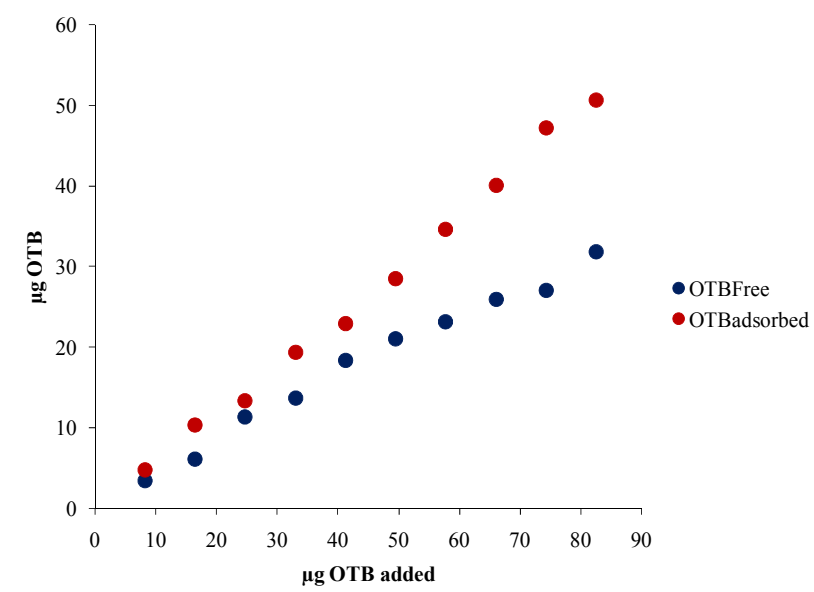

(b)

Figure 7. (a) Absorbance spectra of solutions containing $0.224 \mathrm{mg}$ of Flocculant and increasing additions of 8.26 $\mu \mathrm{g}$ of OTB; (b) Bound and free OTB profile versus total amount of OTB added. 


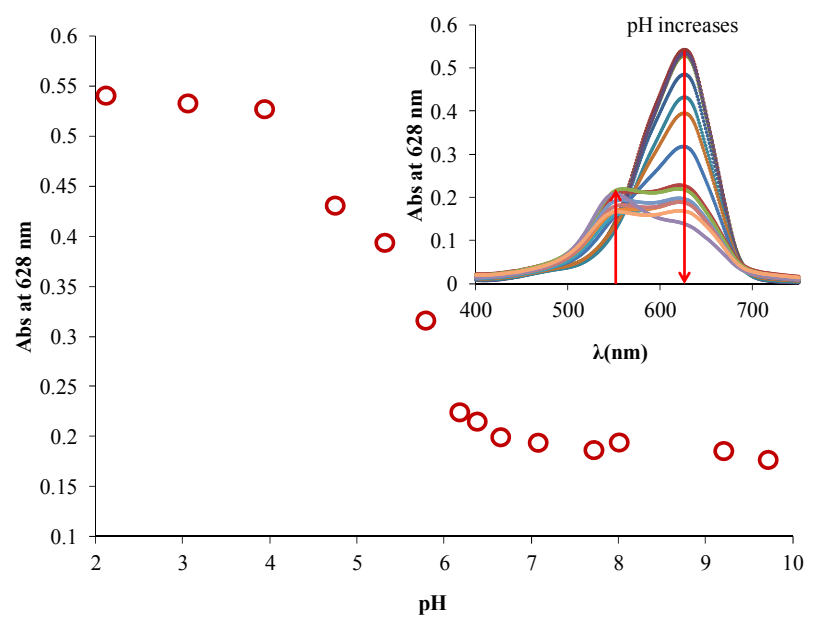

Figure 8. Interaction of OTB-Flocculant vs pH.

culant are not in anionic form and there is not interaction with OTB. As the $\mathrm{pH}$ increases the groups becomes anionic and these favored the formation of Flocculant-OTB complex. Indirectly, this experiment represents the spectroscopic titration of the ionizable groups in Flocculant, which allowed to determinate a pKa 5.45 from these experimental data [26].

In order to elucidate the functional groups in Flocculant, it was analyzed by infrared spectroscopy (Figure 9). The spectra showed significant vibrations at 3338,3185 , 2925 and $1653 \mathrm{~cm}^{-1}$. Considering the experimental data of $\mathrm{pKa}$ and the infrared vibrations is suspected the presence of aliphatic carboxylic groups bearing in the Flocculant.

Moreover, Figure 10 shows the UV-Vis spectra obtained by titration of Flocculant-OTB complex with $\mathrm{Sn}^{2+}$ and $\mathrm{Zn}^{2+}$.

Instead, the absorbance increases gradually with other metal ions reaching the maximum absorbance of OTB at ratios beyond $6.7 \mu \mathrm{g} \mathrm{M}^{\mathrm{n}+} / \mathrm{mg}$ Flocculant (Figure 11).

For example, $14.13 \mu \mathrm{g}(0.048 \mu \mathrm{mol})$ of OTB are released at $2.24 \mu \mathrm{g} \mathrm{Zn} / \mathrm{mg}$ Flocculant ratio, but it continues releasing until $19.01 \mu \mathrm{g}(0.062 \mu \mathrm{mol})$ at $6.7 \mu \mathrm{g}$ $\mathrm{Zn}^{2+} / \mathrm{mg}$ Flocculant. Otherwise, only $8.79 \mu \mathrm{g}(0.029$ $\mu \mathrm{mol})$ of OTB are exchange at $2.24 \mu \mathrm{g} \mathrm{Pb}^{2+} / \mathrm{mg}$ Flocculant. These results indicates the degree of interaction between Flocculant and each metal ion studied, being $\mathrm{Sn}^{2+}$ the one with the most favorable interaction.

In Figure 11 is presented the OTB absorbance profile as function of the titration of Flocculant-OTB complex with metal ions, which is representative of affinity degree between metal ions and Flocculant. The experimental data obtained for $\mathrm{Sn}^{2+}$ indicates has more interaction with Flocculant than other metal ions, due to the fact that a lower dose of this cation was necessary for removal OTB from flocculant-OTB complex. Moreover, for $\mathrm{Sn}^{2+}$ the absorbance decrease for $\mu \mathrm{g}$ metal $/ \mathrm{mg}$ Flocculant $>2.3$ confirming the higher Sn-flocculant affinity. This same behavior occurred in the interaction between aluminum and PVSK relations larger than $17 \mu \mathrm{g}$ metal $/ \mathrm{mg}$ PVSK.

Equations (3) and (4) represent the equilibrium between Flocculant and OTB to form Flocculant-OTB and the equilibrium in which the Flocculant-OTB complex is used to study the physicochemical behavior of the polyelectrolyte by exchange of OTB with metal ions. These two processes are in competition and are associated with equilibrium constants $\mathrm{K}_{3}$ and $\mathrm{K}_{4}$, respectively. The amount of OTB displaced by the metal ion depends on the affinity of the polyelectrolyte toward the cation. The magnitude of the $\mathrm{K}_{4}$ is a relative measure of this affinity. Metal ions which exhibit higher $\mathrm{K}_{4}$ values should effect a greater displacement of OTB.

$$
\begin{aligned}
& \text { Flocculant }(-)+\mathrm{OTB}(+) \\
& \stackrel{\mathrm{K}_{3}}{\rightleftarrows}[\mathrm{OTB}(+) / \text { Flocculant }(-)] \\
& {[\mathrm{OTB}(+) / \text { Flocculant }(-)]+\text { Metal }} \\
& \stackrel{\mathrm{K}_{4}}{\rightleftarrows}[\text { Flocculant }(-) / \text { Metal }]+\mathrm{OTB}_{\text {Free }}
\end{aligned}
$$

The association constant $\mathrm{K}_{3}$ of Flocculant-OTB complex was determined from the absorbance data at $555 \mathrm{~nm}$, while association constants for Flocculant-metal ion complexes were calculated indirectly using absorbance data at $628 \mathrm{~nm}$. Calculation of constants was performed by a curve fitting based on a non-linear least-squares method [26] assuming that each monomeric unit of the polymer reacts with a metal cation forming a 1:1 complex. In Table 2 are shown the association constants values obtained, as we expected the metal ions that performed a greater displacement of OTB in FlocculantOTB complex presented greater magnitudes for $\mathrm{K}_{4}$. Here highlight the $10^{4}$ and $10^{3}$ values for Flocculant- $-\mathrm{Sn}^{2+}$ and Flocculant- $\mathrm{Zn}^{2+}$ complexes respectively, which mean the Flocculant forms a highly stable complexes with these metal ions and it is in agreement with their observed experimental behavior. Moreover, the association constants $\mathrm{K}_{4}$ for rest of metal ions are above of $\mathrm{K}_{3}$ value due to their ability to displace OTB and the affinity of Flucculant for metal ions.

Comparing all results about physicochemical characteristics of PVSK and Flocculant is noteworthy that the interaction PVS-OTB and Flocculant-OTB are much different. In the first one, saturation occurs at very small relationship OTB/PVSK indicating a strong interaction; while with the second one, saturation was not observed even with higher amounts of total OTB added.

Also, metal ion titrations require ratios higher than 20 $\mu \mathrm{g} \mathrm{M}^{\mathrm{n}+} / \mathrm{mg}$ PVSK for maximum displacement of the bound OTB, and with all that is not totally displaced. While Flocculant-metal ion interaction relationships are less than $10 \mu \mathrm{g} \mathrm{M}^{\mathrm{n}+} / \mathrm{mg}$ Flocculant and metal ions with 


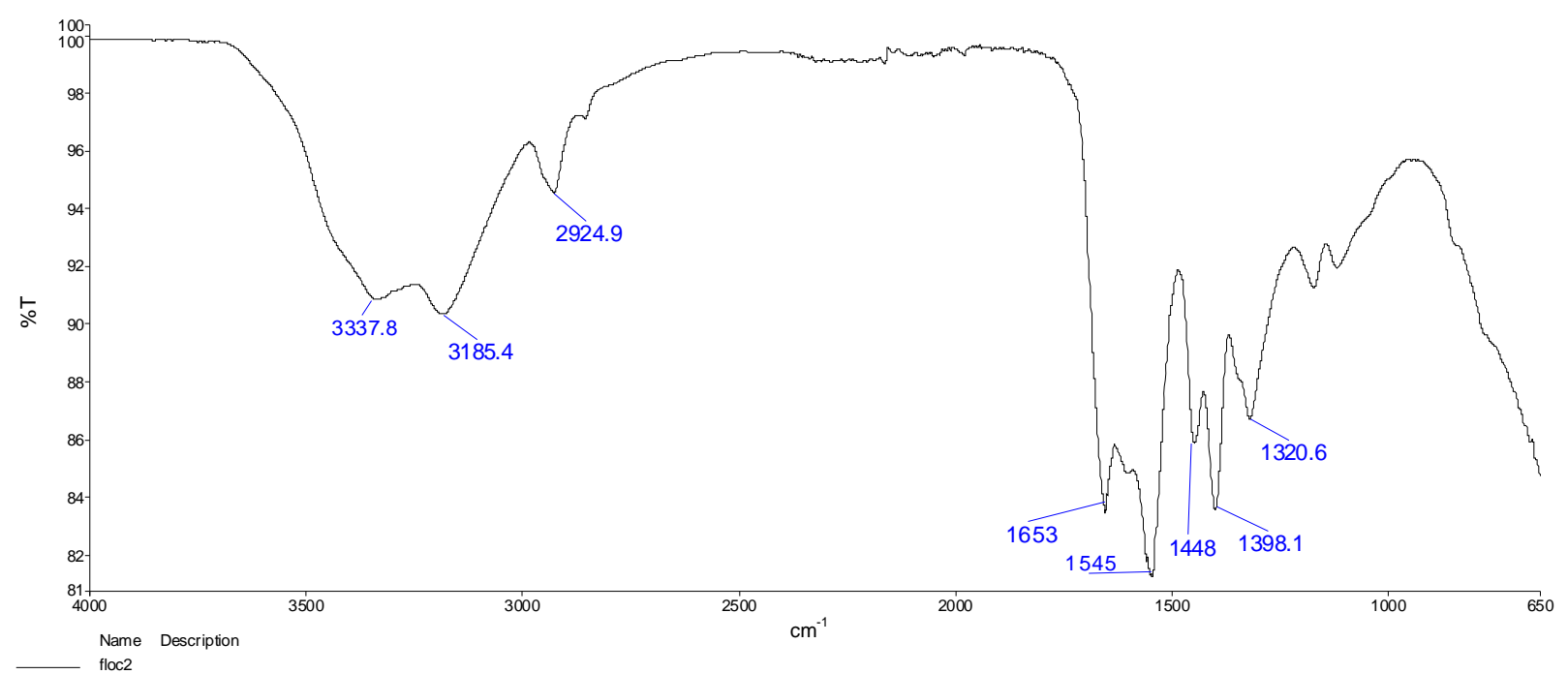

Figure 9. FTIR spectra of Flocculant.

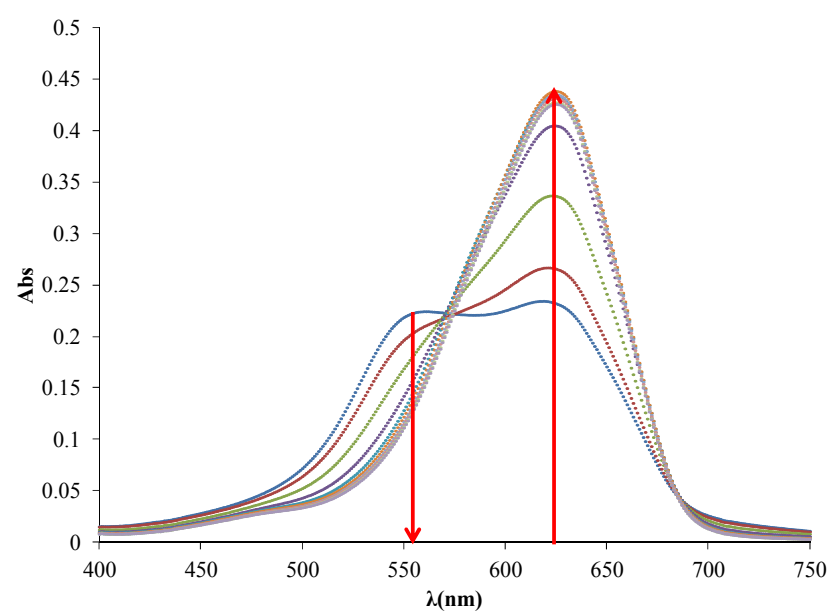

(a)

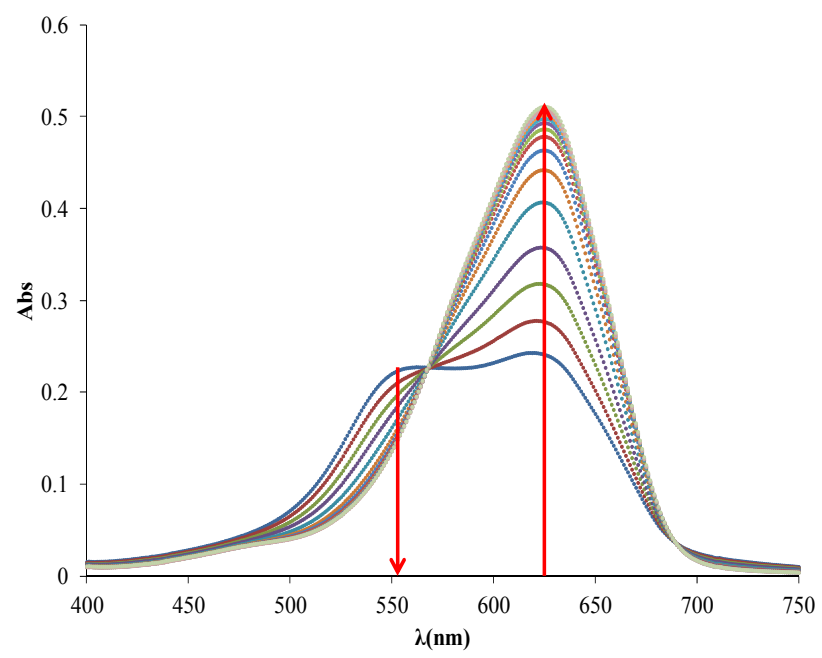

(c)

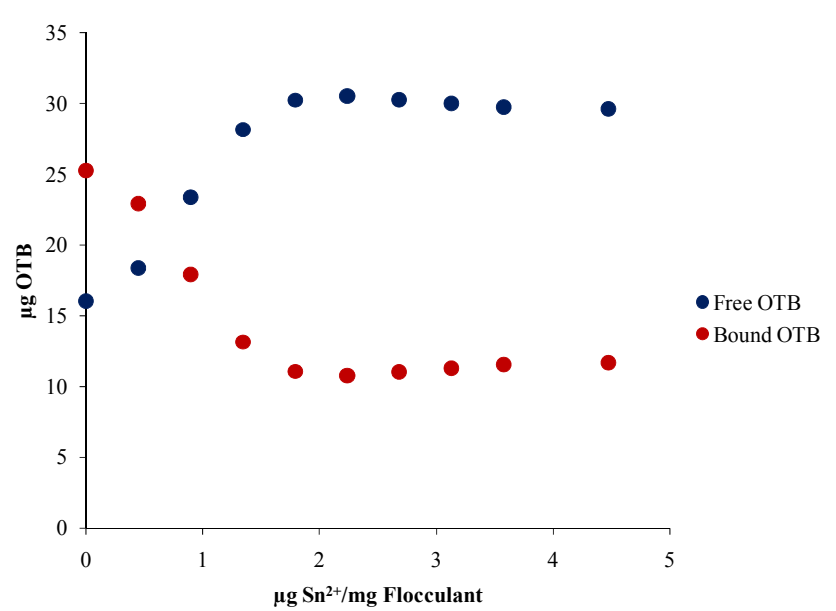

(b)

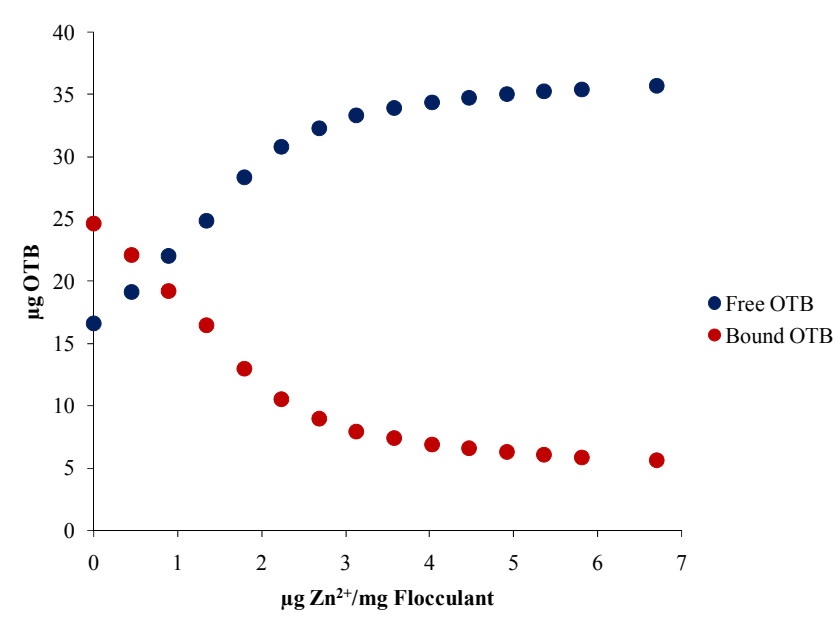

(d)

Figura 10. UV-Vis spectra obtained by titration of Flocculant-OTB complex with $\mathrm{Sn}^{2+}$ and $\mathrm{Zn}^{2+}$ (a) and (c) and bound and free OTB amount profiles (b) and (d). 


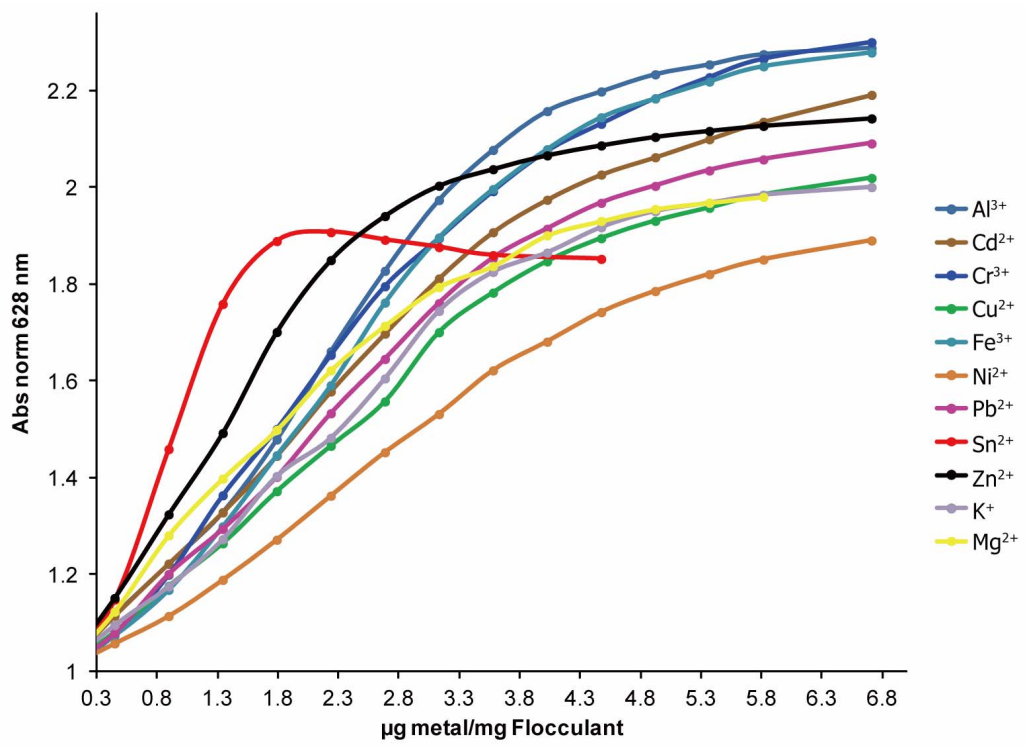

Figure 11. OTB absorbance profiles obtained by titration of Flocculant-OTB complex with metal ions.

Table 2. Relative association constants of Flocculant-metal ion complexes.

\begin{tabular}{cc}
\hline Metal Ion & $\mathbf{K}(\mathbf{m L} / \mathbf{\mu g})$ \\
\hline $\mathrm{Sn}^{2+}$ & 17373.43 \\
$\mathrm{Zn}^{2+}$ & 2730.99 \\
$\mathrm{Mg}^{2+}$ & 1466.92 \\
$\mathrm{~K}^{+}$ & 764.98 \\
$\mathrm{Al}^{3+}$ & 637.96 \\
$\mathrm{Cd}^{2+}$ & 600.67 \\
$\mathrm{Cr}^{3+}$ & 569.14 \\
$\mathrm{~Pb}^{2+}$ & 556.71 \\
$\mathrm{Cu}^{2+}$ & 462.92 \\
$\mathrm{Fe}^{3+}$ & 434.36 \\
$\mathrm{Ni}^{2+}$ & 192.03 \\
$\mathrm{OTB}$ & 0.18 \\
\hline
\end{tabular}

high affinity exchange or displaced almost all the bound OTB. In general, relative association constants for PEmetal ion complexes were higher with the Flocculant in comparison with PVS. These results are in agreement with the observed experimental behavior.

\section{Conclusions}

The physicochemical characteristics of polyelectrolytes as PVSK and Flocculant used in waste water treatment for suspended solids and metal ions removal are significantly different. PVSK is a strong polyelectrolyte and its interaction with OTB and metal ions does not depend of $\mathrm{pH}$. While Flocculant is a weak polyelectrolyte and as was demonstrated in this study, its interaction is favored at neutral or basic conditions. KPVS has great affinity for
$\mathrm{Al}^{3+}$ and $\mathrm{Mg}^{2+}$, hard metal ions commonly associated with suspended solids, this is why is commonly used as coagulant. Flocculant has considerably affinity for various metal ions, but the most important interaction is with $\mathrm{Sn}^{2+}$, which is an important component in residual water of semiconductor industry. Due to this, its performance in waste water treatment is metal ions removal.

In conclusion, in this work was developed a practical methodology in order to evaluate the physicochemical behavior of cationic polyelectrolytes used in the waste water treatment based in PVSK and Flocculant affinity for OTB their mutual affinity for metal ions. UV-Vis spectroscopic titration technique allowed studying these interactions and calculating relative association constants.

\section{Acknowledgements}

Authors acknowledge the enterprise International Rectifiers, S. A., which through Manuel González, Eng., gave a confident vote to a research group situated in Tijuana, to develop a systematic and scientific work that helped for a better operation of their Waste Water Treatment Plant. E. A. López-Maldonado gives his gratitude to the National Council of Science and Technology (CONA$\mathrm{CyT}$ ) in Mexico for the fellowship received in his Ph.D. studies and to Dirección General de Educación Superior Tecnológica (DGEST) for financial support in the development of this research (Grant. No. 4637.12-P).

\section{REFERENCES}

[1] A. E. Hatch, "Process of Treating Mycelia of Fungi for Retention of Metals," US Patent 3859210, 1975.

[2] B. L. Rivas and K. E. Geckeler, "Synthesis and Metal 
Complexation of Poly (Ethyleneimine) and Derivatives," Advances in Polymer Science, Vol. 102, 1992, pp. 171188. doi:10.1007/3-540-55090-9 6

[3] H. F. Morawetz, "Specific Ion Binding by Polyelectrolytes," Fortschr. Hochpolym.-Forsch, 1958, pp. 1-34.

[4] R. D. Porasso and J. C. Benegas, "Chemical Bonding of Divalent Counterions to Linear Polyelectrolytes: Theoretical Treatment within the Counterion Condensation Theory," Physical Chemistry Chemical Physics, Vol. 3, No. 6, 2001, pp. 1057-1062. doi:10.1039/b008401h

[5] M. A. G. T. van den Hoop and J. C. Benegas, "Colloids and Surfaces A: Physicochemical and Engineering Aspects," Colloids and Surfaces A: Physicochemical and Engineering Aspects, Vol. 170, No. 2-3, 2000, pp. 151160.

[6] R. D. Porasso, J. C. Benegas and M. A. G. T. van den Hoop, "Chemical and Electrostatic Association of Various Metal Ions by Poly (Acrylic Acid) and Poly (Methacrylic Acid) as Studied by Potentiometry," The Journal of Physical Chemistry B, Vol. 103, No. 13, 1999, pp. 23612365. doi:10.1021/ip9839706

[7] M. P. Fong, K. Pradeep and T. T. Tjoon, "Removal of Lead, Zinc and Iron by Coagulation-Flocculation," Journal of the Taiwan Institute of Chemical Engineers, Vol. 42, No. 5, 2011, pp. 809-815. doi:10.1016/j.jtice.2011.01.009

[8] M. Borkovec, G. J. M. Koper and C. Piguet, "Ion Binding to Polyelectrolytes," Current Opinion in Colloid \& Interface Science, Vol. 11, No. 5, 2006, pp. 280-289. doi:10.1016/j.cocis.2006.08.004

[9] B. Chaufer and A. Deratani, "Removal of Metal Ions by Complexation-Ultrafiltration Using Water-Soluble Macromolecules: Perspective of Application to Wastewater Treatment," Nuclear and Chemical Waste Management, Vol. 8, No. 3, 1987, pp. 175-187. doi:10.1016/0191-815X(88)90025-3

[10] H. H. G. Jellinek and S. P. Sangal, "Complexation of Metal Ions with Natural Polyelectrolytes (Removal and Recovery of Metal Ions from Polluted Waters)," Water Research, Vol. 6, No. 3, 1972, pp. 305-314. doi:10.1016/0043-1354(72)90008-5

[11] H. P. Gregor, L. B. Luttinger and E. M. Loebl, "MetalPolyelectrolyte Complexes. I. The Polyacrylic AcidCopper Complex," The Journal of Physical Chemistry, Vol. 59, No. 1, 1955, pp. 34-39. doi:10.1021/j150523a011

[12] A. M. Kotliar and H. Morawetz, "Chelation of Copper(II) with Polyacrylic and Polymethacrylic Acid," Journal of the American Chemical Society, Vol. 77, No. 14, 1955, pp. 3692-3695.. doi:10.1021/ja01619a005

[13] H. Nishikawa and E. Tsuchida, "Complexation and Form of Poly (Vinylpyridine) Derivatives with Copper(II) in Aqueous Solution," The Journal of Physical Chemistry, Vol. 79, No. 19, 1975, pp. 2072-2076. doi:10.1021/j100586a018

[14] J. C. Leyte, L. H. Zuiderweg and M. V. Reisen, "Spectroscopic Study of Binuclear Copper Complexes in Aqueous Poly (Methacrylic Acid) Solutions," The Journal of Physi- cal Chemistry, Vol. 72, No. 4, 1968, pp. 1127-1132. doi:10.1021/j100850a007

[15] H. S. Yokoi and I. M. Kawata, "Interaction Modes between Heavy Metal Ions and Water-Soluble Polymers. 2. Spectroscopic and Magnetic Reexamination of the Aqueous Solutions of Cupric Ions and Poly (Acrylic Acid)," Journal of the American Chemical Society, Vol. 108, No. 12, 1986, pp. 3361-3365. doi:10.1021/ja00272a034

[16] A. M. Liquorio, F. Ascoli and C. Botré, "On the Electrostatic Interactions between Counterions and Macroions in Polyelectrolyte Solutions," Journal of Polymer Science, Vol. 40, No. 136, 1959, pp. 169-178. doi:10.1002/pol.1959.1204013613

[17] G. De Jong Hans, J. Lyklema and H. P. van Leeuwen, "Conductometric Analysis of the Competition between Monovalent and Divalent Counterions in Their Interaction with Polyelectrolytes," Biophysical Chemistry, Vol. 27, No. 2, 1987, pp. 173-182. doi:10.1016/0301-4622(87)80056-X

[18] L. R. Bernabé, N. Schiappacasse and L. A. Basáez, "Polyelectrolyte-Cu(II) Interactions Studied by Cyclic Voltammetry," Polymer Bulletin, Vol. 45, No. 3, 2000, pp. 259-265. doi:10.1007/s002890070029

[19] S. Peng and C. Wu, "Light Scattering Study of the Formation and Structure of Partially Hydrolyzed Poly(Acrylamide)/Calcium(II) Complexes," Macromolecules, Vol. 32, No. 3, 1999, pp. 585-589. doi:10.1021/ma9809031

[20] Y. Zhang, J. F. Douglas and B. D. J. Ermi, "Influence of Counterion Valency on the Scattering Properties of Highly Charged Polyelectrolyte Solutions," Chemical Physics, Vol. 114, No. 7, 2001, pp. 3299-3313. doi: $10.1063 / 1.1336148$

[21] L. R. Bernabé, S. A. Pooley and E. Pereira, "WaterSoluble Polymer Materials as Complexing Reagents for the Separation of Metal Ions Using Membrane Filtration," Polymers for Advanced Technologies, Vol. 17, No. 11-12, 2006, pp. 865-871. doi:10.1002/pat.791

[22] L. R. Bernabé, E. Pereira and I. M. Villoslada, "WaterSoluble Polymer-Metal Ion Interactions," Progress in Polymer Science, Vol. 28, No. 2, 2003, pp. 173-208. doi:10.1016/S0079-6700(02)00028-X

[23] Z. Iatridi and G. Bokias, "Formation of Ternary Poly (Acrylic Acid)-Surfactant- $\mathrm{Cu}^{2+}$ Complexes in Aqueous Solution: Quenching of Pyrene Fluorescence and $\mathrm{pH}-$ Controlled 'On-Off' Emitting Properties," Langmuir, Vol. 24, No. 20, 2008, pp. 11506-11513. doi:10.1021/la8019793

[24] I. Nagata and Y. Okamoto, "Investigation on Ion Binding in Synthetic Polyelectrolyte Solutions Using Rare Earth Metal Fluorescence Probes," Macromolecules, Vol. 16, No. 5, 1983, pp. 749-753. doi:10.1021/ma00239a009

[25] E. A. López-Maldonado, A. Ochoa-Terán and M. T. Oropeza, "A Multiparameter Colloidal Titrations for the Determination of Cationic Polyelectrolytes," Journal of Environmental Protection, Vol. 3, 2012, pp. 1559-1570.

[26] M. Inoue, "Excel ${ }^{\circledR}$ Worksheets for Spectrometry," Universidad de Sonora, Hermosillo, 2009. 